\title{
Special Issue 2007 in Natural Hazards on "Modelling and simulation of dangerous phenomena, and innovative techniques for hazard mapping and mitigation"
}

\author{
Giulio G. R. Iovine • Michael F. Sheridan
}

Received: 5 February 2009/Accepted: 5 February 2009/Published online: 4 July 2009

(C) Springer Science+Business Media B.V. 2009

Hazard mapping and mitigation approaches take advantage of a number of different techniques of analysis, based on empirical evaluations of causal factors, historical investigations and statistical analyses of past events, numerical models (which range in type from simplified to physically based), laboratory experiments, and theoretical studies. Recently, the availability of powerful computing environments and of GIS allowed to effectively handle increasing amounts of data and to develop quantitative techniques of analysis for hazard evaluation and management.

The need for coping with hazards is underlined by the long list of severe damaging events of different type, which affected different zones of the planet even in the past few months. Even when restricting the view to one single quite small country, the series of natural disasters appears to be undoubtedly excessive. For instance, in early May 2009-at the time this Preface is being written-Calabria (Southern Italy) has just come out from an exceptional rainy period, which lasted from November to March and triggered numerous landslides, erosive processes, and flooding. At the end of January, two men were killed by a debris flow which impacted on the A3 highway, and few other persons were injured: nevertheless, the effects induced by the rainfall had been even worse, if only eventful circumstances had not helped. Starting from the end of January, also medium-sized slope movements began to mobilise, in certain cases inducing severe damage to urbanised areas, cemeteries, and lifelines. Meantime, a medium-intensity seismic swarm devastated the surroundings of L'Aquila, in Abruzzo (Central Italy), culminating in the earthquakes of 6 and 7 April, characterised by $M=5.8$ and 5.3, respectively, and causing about 300 victims. The real causes of such a disproportionate toll of human lives are still being investigated in detail; though, in addition to obvious local ground factors, from the very

G. G. R. Iovine $(\bowtie)$

CNR-IRPI, National Research Council, Research Institute for Geo-Hydrologic Protection,

Via Cavour 6, 87036 Rende, Cosenza, Italy

e-mail: g.iovine@irpi.cnr.it

M. F. Sheridan

Center for GeoHazards Studies, University at Buffalo, 411 Cooke Hall,

SUNY Buffalo, NY 14260, USA

e-mail: mfs@geology.buffalo.edu 
beginning several sins appeared to have diffusely been committed by the different protagonists of this event (designers, building firms, public administrators, individuals) for a long time. Finally, in the past hours, heavy rains are again striking the country. In the NW, floods are threatening lifelines, bridges, and urbanised sectors along the Tanaro River (in Piedmont) since 28 April, thus obliging the National Department for Civil Protection to manage a new territorial crisis. Needless to say, most Italians completely repressed the memory of a severe and prolonged drought suffered only few months ago, in the SpringSummer of 2008 .

The set of manuscripts collected in the present special issue originated as a selection of studies presented - either as oral talks or posters-at conferences convened in 2007 in Wien (EGU, session NH11.04), Bangkok (AOGS, session IWG02), and Rimini (Geoitalia, session T34).

When the call for papers was issued, a total number of 15 manuscripts were submitted for publication in this special issue, covering different topics related to the general theme here considered. All the manuscripts then underwent a thorough phase of review, which consisted of two or more rounds of evaluations.

It is perhaps useful to add here few considerations on our experience of guest-editing. Even though many Authors and Reviewers interpreted their role in all seriousness, surprisingly some of the texts were insufficiently structured, written in poor English, and supplied with low-quality figures and obscure tables with unsatisfying legends and captions. The state of the art of the considered topics was not commented adequately, as well as references, and strong and weak points of mentioned approaches were not discussed. Too often, the attempts of modelling and hazard mapping had not been validated against real cases, and calibration and sensitivity analyses were missing. In addition, quite irritating habits are becoming too much widespread: it is in fact common to find the same results published several times, either in journals or proceedings, even repeating entire periods and figures. In other cases, entire portions of publications written by other scientists are merely copied and pasted, even without any referencing. Among the most impressive cases, some authors submitted a manuscript which was the identical copy of another already published in the proceedings of an international conference.

At the end of the review phase, 12 manuscripts were accepted, two were rejected, and one was withdrawn. Among the accepted manuscripts, the first four deal with problems related to flooding and inundation; the following three manuscripts concern machine learning methods, seismicity, and volcanic processes, respectively; the remaining five manuscripts analyse slope-movement-related problems.

More in detail, $\mathrm{Li}$ et al. present a numerical model designed to study the storm surge induced by typhoon along the coast of Taiwan, and test it against the storm surges induced by Typhoons Herb in 1996 and by typhoons Kai-Tak and Bilis in 2000.

El Kadi Abderrezzak et al. describe an attempt of 2-D numerical modelling of flash flood propagation in urban areas after an excessive rainfall event or dam/dyke break wave. Four applications are described, ranging from laboratory experiments to real cases occurred in Italy, France, and Spain.

Calvo and Savi propose an adaptive, conceptual model for real-time flood forecasting, able to simulate rainfall-runoff transformations and to reproduce the contributions of numerous ungauged sub-basins of the Tiber river catchment (central Italy). The model is tested under real-time forecasting conditions for three historical flood events, and the obtained $12 \mathrm{~h}$ of lead time appears to be useful for civil protection purposes.

Di Baldassarre et al. propose a method for producing probability-weighted flood hazard maps, based on combined 1-D and 2-D numerical simulations, with the aim of comparing 
different strategies of risk management for a reach of the River Reno (northern-central Italy).

Pozdnoukhov et al. discuss appropriate tools to process large amounts of data, gathered through automatic monitoring networks, aiming at predicting hazard map at fine spatial scales. At this purpose, they adopt algorithms of machine learning to produce prediction maps in real time, useful in risk assessment and mitigation, with examples of application in Switzerland.

Georgoudas et al. describe a 2-D Cellular Automata model for the simulation of the earthquake process, calibrated against real cases occurred in the Xanthi area (north-eastern Greece) from 1981 to 2004, and its on-chip implementation based on Field Programmable Gate Array logic. The response of the processor provides the Gutenberg-Richter scaling law of the area under study. The hardware implementation of the model allows low cost, high speed, compactness, and portability features.

Vicari et al. present an automated system employing thermal-infrared satellite MODIS data to estimate time-averaged rates of lava discharge. Authors use the time-varying discharge rates obtained for the July 2006 eruption of Mt. Etna (Sicily, southern Italy) for driving lava flow simulations with a Cellular Automata model, and show how this approach can be adopted during an ongoing eruptive event.

Falaschi et al. discuss a multidisciplinary approach to landslide susceptibility mapping based on Logistic Regression, Artificial Neural Network, and GIS, with an example of application in the middle reach of the River Serchio (Tuscany, central Italy). The application of a multilayer Neural Network, equipped for supervised learning and error control, permits to improve performance of the model.

Jiménez-Perálvarez et al. describe a method for landslide susceptibility analysis, mapping, and validation, based on a GIS Matrix Method. The approach is validated by verifying the degree of fit between a set of real landslides (not used for the susceptibility analysis) and the obtained susceptibility map for a study area in the Sierra Nevada-Betic Cordillera (Spain).

D'Amato Avanzi et al. present a method for soil slip susceptibility assessment in the Cardoso Torrent (Apuan Alps, central Italy) hit in June 1996 by an extreme rainstorm which caused victims and destruction. The adopted approach couples a mechanical model (based on an infinite-slope form of the Mohr-Coulomb failure criterion) and a steady-state hydrological model (which considers the cohesion of the material potentially involved in landsliding). The effects of topographic convergence and drainage area on slope failure are evaluated by means of GIS techniques.

Scotto di Santolo and Evangelista discuss the problem of the prediction of dynamic parameters of debris flows in pyroclastic deposits, with back-analysis of real cases from Campania (southern Italy) based on two different rheological models (Voellmy and frictional).

Finally, Lin et al. analyse motion and deposition characteristics of debris flows, and their relationship with land-use planning and management. A friction-collision rheological model is tested against laboratory results and real events. They find that topography of the channel bed, yield stress level of the debris flows, and inflow pattern have significant influence on the simulated flow motion and deposition characteristics.

The papers included in the present issue underline the complexity and challenges still to be faced in the considered field of research and the need of further scientific effortshopefully fed by adequate financial support. 